\title{
Unilateral Complete Ptosis after Scalp Block for Awake Craniotomy: A Rare Complication
}

\author{
Mohamad Hasyizan Hassan ${ }^{1}$ Wan Mohd Nazaruddin Wan Hassan ${ }^{1}$ Regunath Kandasamy ${ }^{2}$ \\ Soon Eu Chong ${ }^{3}$
}

\author{
${ }^{1}$ Department of Anaesthesiology and Intensive Care, School of \\ Medical Sciences, Health Campus, Universiti Sains Malaysia, \\ Kelantan, Malaysia \\ ${ }^{2}$ Department Neuroscience, School of Medical Sciences, Health \\ Campus, Universiti Sains Malaysia, Kelantan, Malaysia \\ ${ }^{3}$ Regenerative Medicine Cluster, Advanced Medical and Dental \\ Institute, Universiti Sains Malaysia, Penang, Malaysia \\ J Neuroanaesthesiol Crit Care 2018;5:111-113.
}

\begin{abstract}
Address for correspondence Mohamad Hasyizan Hassan, MBBS, MMed (anesth), Department of Anaesthesiology and Intensive Care, School of Medical Sciences, Health Campus, Universiti Sains Malaysia, 16150 Kubang Kerian, Kelantan, Malaysia (e-mail: miezan82@yahoo.com, hasyizan@usm.my).
\end{abstract}

\begin{abstract}
A combination of scalp block and monitored anesthesia care (MAC) is one of the options among anesthetic techniques that can be used during an awake craniotomy for epilepsy surgery. Even though a scalp block is useful as locoregional analgesia during the surgery, it also has the potential to cause some complications. Trigeminocardiac Keywords

- awake craniotomy

- scalp block

- ptosis

- ropivacaine reflex and transient facial nerve palsies have previously been reported following scalp block. The toxicity of local anesthetic agents and nerve injuries present other potential complications. However, complete unilateral ptosis is a rare complication after scalp block. We report a case of unilateral complete mechanical ptosis after a scalp block using ropivacaine $0.75 \%$ for an awake craniotomy for epilepsy surgery.
\end{abstract}

\section{Case Report}

A 14-year-old, 55-kg girl with a known case of epilepsy with complex partial seizures was electively admitted for a right craniectomy and electrocorticography (ECoG) electrode insertion. She was diagnosed with epilepsy secondary to a right temporal dysembryoplastic neuroepithelial tumor (DNET). On preoperative assessment, she was asymptomatic and had been free from seizure episodes for approximately 1 month with oral carbamazepine $300 \mathrm{mg}$ OM and $400 \mathrm{mg}$ ON. On examination, the patient was fully alert, conscious, and cooperative, with normal higher mental function. There were no cranial nerve palsies or other focal neurologic signs. The assessment of the motor, sensory, and cerebellar systems was also unremarkable.

The patient underwent two consecutive phases of surgeries. The first surgery, for the right temporoparietal craniectomy and the insertion of ECoG electrodes, was performed under general anesthesia. The surgery was uneventful, and the patient was extubated immediately after surgery. The second surgery, for reopening of the scalp, ECoG electrode removal, lesionectomy, and cranioplasty were performed 3 days after the first operation, under awake craniotomy with monitored anesthesia care (MAC) and a scalp block.

The scalp block was performed using ropivacaine 0.75\% with $5 \mu \mathrm{g} / \mathrm{mL}$ adrenaline. A volume of 2.5 to $3.0 \mathrm{~mL}$ of ropivacaine $0.75 \%$ was infiltrated at each nerve that innervated the bilateral scalp-the supratrochlear, supraorbital, zygomaticotemporal, auriculotemporal, lesser occipital, and greater occipital nerves-making up a total volume of $30 \mathrm{~mL}$ of ropivacaine $0.75 \%$ for 12 sites of infiltration bilaterally. The block of left supraorbital nerve was performed subcutaneously at left supraorbital notch with the needle pointed perpendicularly during 2.5 to $3.0 \mathrm{~mL}$ injection of local anesthesia. After that, the needle was directed medially along the orbital wall border as far as the bridge of the nose to block left supratrochlear nerve with another 2.5 to $3.0 \mathrm{~mL}$ of local anesthesia given as a subcutaneous sausage. The total local anesthetic dose given for the whole block did not exceed the maximum allowable dose of $4 \mathrm{mg} / \mathrm{kg}$.

A loading dose of intravenous (IV) dexmedetomidine at $1 \mu \mathrm{g} / \mathrm{kg}$ over 10 minutes was administered while performing received

February 25, 2018

accepted

May 28, 2018

published online

June 23, 2018
DOI https://doi.org/

10.1055/s-0038-1665545

ISSN 2348-0548.
Copyright $\odot 2018$ Indian Society of Neuroanaesthesiology and Critical Care
License terms

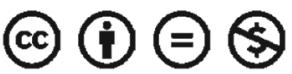


the scalp block infiltration, with subsequent maintenance of $0.7 \mu \mathrm{g} / \mathrm{kg} / \mathrm{h}$ (B. Braun Perfusor Space, Firma Braun, Melsungen, Germany), combined with an IV infusion of remifentanil at 0.05 to $0.1 \mu \mathrm{g} / \mathrm{kg} / \mathrm{min}$ (B. Braun Perfusor Space) and a target-controlled infusion of propofol at $1.5 \mu \mathrm{g} / \mathrm{mL}$ (Alaris PK pump, Paedfusor model, CareFusion, Yorba Linda, California, United States). The BIS (bispectral index monitor) was kept around 70 to 100 throughout the operation. After head pinning and positioning, sedation was maintained only by a low dose of dexmedetomidine $(0.2 \mu \mathrm{g} / \mathrm{kg} / \mathrm{h})$ to achieve an adequate conscious sedation effect.

Intraoperatively, the patient was comfortable, cooperative, and arousable, but complete left eye ptosis began to be noticed during the operation. The left eye ptosis gradually worsened and complete ptosis was observed approximately 30 minutes after the injection of the scalp block. However, there were no associated airway obstructive symptoms, and the vital signs were normal. There was no focal neurological deficit nor any intraoperative episode of fit. The surgery ended after 5 hours, and the patient did not complain of any pain during or after the operation.

Postoperatively, the full neurological assessment was repeated. There was no associated diplopia, and the extraocular muscle movements were intact. The visual field and visual acuity were normal as well. The pupillary size was equal and reactive in both the eyes, and there was no other cranial nerve abnormality detected. The motor function of muscles for facial expression was normal. The higher mental functions, as well as the motor, sensory, and cerebellar systems, were otherwise intact following the operation. The patient was subsequently monitored in the neurocritical care unit, and a referral to the ophthalmology team was made. Reassurance was given to the patient, and no steroid was prescribed.

The complete left eye ptosis gradually improved to $75 \%$ ptosis after day 1 , and total resolution of the ptosis was seen on day 2. A diagnosis of complete left eye mechanical ptosis secondary to local anesthetic injection was made. The patient was reassured, and she was subsequently discharged on the fourth day after the operation.

\section{Discussion}

Awake craniotomy is the favored technique of neurosurgery for certain procedures such as tumor excision near the eloquent cortex, epilepsy surgery, and deep brain stimulation surgery. The MAC technique with a scalp block as locoregional analgesia is one of the anesthetic options for this surgery, aside from the asleep-awake-asleep technique.

A scalp block offers adequate analgesia during an awake craniotomy for smoothness of intraoperative cortical mapping and neurological assessment; at the same time, it provides hemodynamic stability and stress response reduction. ${ }^{1}$ A scalp block using adrenaline containing local anesthetic of ropivacaine and levobupivacaine, with the dosage up to $3.6 \mathrm{mg} / \mathrm{kg}$ and $2.5 \mathrm{mg} / \mathrm{kg}$, respectively, has been proven to be safe, despite the rapid increase in the plasma level. ${ }^{2}$ The local anesthetic is infiltrated into the auriculotemporal nerve, zygomaticotemporal nerve, supratrochlear nerve, supraorbital nerve, the great auricular nerve, and the greater and lesser occipital nerves. Targeted infiltration of 2 to $3 \mathrm{~mL}$ of local anesthetic at multiple sites with a 23 - to 25 -gauge needle is commonly used during the scalp block. ${ }^{3}$ Alternatively, a superficial cervical plexus block may reduce the amount of local infiltration of the scalp block. ${ }^{4} \mathrm{~A}$ combination of remifentanil and dexmedetomidine infusions is used to provide rapid-onset, rapid-offset, and good analgesia with anxiolysis and conscious sedation. Complications of awake craniotomies include seizures, bleeding, nausea, vomiting, obstructive apnea, hypertension, air embolisms, hypoventilation, tachycardia, and loss of patient cooperation.

The trigeminocardiac reflex can be a result of a scalp block or manipulation of the dura. ${ }^{5,6}$ This reflex is caused by afferent fibers arising from sensory branches of the trigeminal nerve causing stimulation of cardiac branches of the vagus nerve. The rapid infiltration of the local anesthetic on the supratrochlear nerve may induce compression and stretching in the nerve and provoke the reflex. ${ }^{6}$ An incident of transient facial nerve palsy due to direct injury to auriculotemporal nerve block has also been reported. ${ }^{7}$ Nevertheless, the incidence of unilateral complete ptosis has been extremely low.

Mechanical ptosis can be caused by excessive local anesthetic infiltration causing injury or edema in the muscles responsible for eyelid retraction. In contrast, facial nerve palsy secondary to auriculotemporal nerve injury leads to depression of the eyebrow due to dysfunction of the frontalis muscle. The levator palpebrae superioris, Müller's muscle, and frontalis muscle are responsible for eyelid retraction. The former, which is the predominant muscle, originates from the lesser wing of the sphenoid bone and traverses the orbit; it may be injured due to local anesthetic infiltration. In this case, the absence of Horner's syndrome and the presence of normal extraocular muscle movements exclude a diagnosis of neurogenic ptosis. Aponeurotic ptosis was also impossible because the area of surgery, clamping, and skin incision was distant from the orbit and the eyelid.

Although the incidence of local anesthetic toxicity is extremely low, caution should always be used during a scalp block because the scalp is highly vascular and potential adverse cardiovascular and central nervous system side effects can occur as early as 5 minutes after infiltration. In practice, most clinicians use 3 to $5 \mathrm{~mL}$ of local anesthetic volume at each nerve. ${ }^{3,7}$, However, the volume of local infiltrates in pediatric patients should be revised. Costello and Cormack $^{9}$ suggested a maximum volume of $40 \mathrm{~mL}$ of ropivacaine $0.75 \%$ with adrenaline $1: 200,000$ dilution for a 70 - to $90-\mathrm{kg}$ adult. As a wide therapeutic window between clinical efficacy and adverse reaction is seen in both levobupivacaine and ropivacaine, these drugs were preferentially used over bupivacaine for scalp blocks. ${ }^{2}$

In conclusion, attention to the anatomical landmarks and correct techniques during local anesthetic injections on the supratrochlear and supraorbital nerve, together with a judiciously chosen volume of infiltrates, is crucial to prevent iatrogenic ptosis. 


\section{Funding}

None.

\section{Conflict of Interest}

None.

\section{References}

1 Burnand C, Sebastian J. Anaesthesia for awake craniotomy. Contin Educ Anaesth Crit Care Pain 2013;14(1):6-11

2 Costello TG, Cormack JR, Mather LE, LaFerlita B, Murphy MA, Harris K. Plasma levobupivacaine concentrations following scalp block in patients undergoing awake craniotomy. $\mathrm{Br} \mathrm{J}$ Anaesth 2005;94(6):848-851

3 Guilfoyle MR, Helmy A, Duane D, Hutchinson PJ. Regional scalp block for postcraniotomy analgesia: a systematic review and meta-analysis. Anesth Analg 2013;116(5):1093-1102
4 Bonhomme V, Franssen C, Hans P. Awake craniotomy. Eur J Anaesthesiol 2009;26(11):906-912

5 Prabhu VC, Bamber NI, Shea JF, Jellish WS. Avoidance and management of trigeminocardiac reflex complicating awakecraniotomy. Clin Neurol Neurosurg 2008;110(10):1064-1067

6 Chowdhury T, Baron K, Cappellani RB. Severe bradycardia during scalp nerve block in patient undergoing awake craniotomy. Saudi J Anaesth 2013;7(3):356-357

7 McNicholas E, Bilotta F, Titi L, Chandler J, Rosa G, Koht A. Transient facial nerve palsy after auriculotemporal nerve block in awake craniotomy patients. A A Case Rep 2014;2(4):40-43

8 Pinosky ML, Fishman RL, Reeves ST, et al. The effect of bupivacaine skull block on the hemodynamic response to craniotomy. Anesth Analg 1996;83(6):1256-1261

9 Costello TG, Cormack JR. Anaesthesia for awake craniotomy: a modern approach. J Clin Neurosci 2004;11(1):16-19 